\title{
Cesium-137 Gamma Radiation
}

National Cancer Institute

\section{Source}

National Cancer Institute. Cesium-137 Gamma Radiation. NCI Thesaurus. Code C129643.

Gamma rays produced by the decay of cesium- 137 . 